\title{
Additive Manufacturing of Ni-Mn-Cu-Ga: Influence of Sintering Temperature on Magnetocaloric Effect and Microstructure
}

\author{
Erica Stevens ${ }^{1}$, Daniel Salazar ${ }^{2}$, Katerina Kimes ${ }^{1}$, Rafael Rodriguez de Vecchis ${ }^{1}$, Volodymyr \\ Chernenko $^{2,3}$, and Markus Chmielus ${ }^{1 *}$ \\ 1. Dept. of Mechanical Engineering and Materials Science, Univ. of Pittsburgh, Pittsburgh, PA, USA \\ 2. BCMaterials \& University of the Basque Country (UPV/EHU), 48940 Leioa, Spain \\ 3. Ikerbasque, Basque Foundation for Science, 48013 Bilbao, Spain \\ * Corresponding author: chmielus@ @itt.edu
}

$\mathrm{Cu}$-doped Ni-Mn-Ga exhibits the magnetocaloric effect (MCE): a sharp isothermal change in magnetic entropy and adiabatic temperature change, $\Delta \mathrm{T}_{\mathrm{ad}}$, associated with a change in magnetic field. This magnetic entropy change arises because of a magneto-structural martensitic phase transformation between the high-temperature cubic austenite and tetragonal or orthorhombic martensite phase [1]. Conventional MCE materials like Ni-Mn-Cu-Ga heat up in an applied magnetic field and can be used in a heat pump for solid-state magnetic refrigerators.

Magnetic refrigeration is achieved through a magnetocaloric material-based heat pump, removing heat via fluid flow. This heat exchange requires a magnetocaloric solid with high surface-to-volume ratio, and a low pressure drop in the fluid across the magnetocaloric material [2]. High efficiency magnetic refrigeration may be achieved by producing magnetocaloric heat exchangers with additive manufacturing (AM), allowing for freedom of internal/external geometries, as well as composition/ property gradient structures. Many AM methods utilize full melting, selectively melting powder layerby-layer. Though these full melting techniques show potential for magnetocalorics [3,4], binder jet printing (BJP) has some distinct advantages. In BJP, binder is selectively deposited layer-by-layer onto a powder bed to bind powder particles together, with subsequent curing and sintering for binder burn-out and densification. This process has been found viable for similar composition Ni-Mn-Ga [5].

Samples were fabricated from powder using an ExOne Lab binder jetting 3D printer and sintered in an argon-purged vacuum atmosphere at $1050{ }^{\circ} \mathrm{C}, 1070{ }^{\circ} \mathrm{C}$, and $1080{ }^{\circ} \mathrm{C}$ for $2 \mathrm{~h}$ then air cooled. Characterization was conducted with a Lakeshore 747 VSM, Pyris 6 DSC, and Zeiss Sigma500 FESEM.

Fig. 1 shows the $\Delta \mathrm{T}_{\mathrm{ad}}(\mathrm{T})$ (each point measured at $2 \mathrm{~T}$ magnetic field change) and DSC results for the sintered samples. $\Delta \mathrm{T}_{\mathrm{ad}}$ results obtained on cooling show a decrease in maximum $\Delta \mathrm{T}_{\mathrm{ad}}$ temperature and in transformation hysteresis with increasing sintering temperature. Similarly, in the thermal transformation data, increasing sintering temperature causes the peak to narrow. These two narrowing effects are a result of improved homogenization and grain refinement with the higher sintering temperature.

Micrographs from each sintering condition are given in Fig. 2. The $1050{ }^{\circ} \mathrm{C}$ sample has low bulk density and limited necking/coalescence between particles (Fig. 2a,d). At this joining of particles, spots are observed that were identified via EDS as Mn-O. The $1070{ }^{\circ} \mathrm{C}$ sample (Fig. 2b,e) is similar, with higher bulk density and more coalescence, but still having Mn-O at boundaries. The $1080{ }^{\circ} \mathrm{C}$ sample (Fig. 2c,f ) is more dense and significant grain growth is observed, yet still shows Mn-O particles and holes. Preliminary EDS and XPS results indicate that these particles are either $\mathrm{MnO}$ or $\mathrm{Mn}_{3} \mathrm{O}_{4}$ [6]. Despite presence of oxides, MCE results are very encouraging for the further advancement of this material. 
References:

[1] V Franco et al., Progress in Materials Science 93 (2018), p. 112-232.

[2] A Waske et al., MRS Bulletin 43 (2018), p. 269-273.

[3] E Stevens et al., Microscopy and Microanalysis 24 (2018), p. 956-957.

[4] J Moore et al., Journal of Applied Physics 114 (2013), p. 1-9.

[5] A Mostafaei et al., Acta Materialia 154 (2018), p. 355-364.

[6] The authors acknowledge facilities at the Univ. of Pittsburgh: PINSE NFCF, MMCL, MCL, ANSYS AMRL. E.S. was supported by the DoD through the NDSEG program. K.K. was supported by PPG and SSOE. Project MAT2014-56116-C4-1-3-4-R from MINECO, Spain is acknowledged. A Perez, J

Feuchtwanger, A Mostafaei, A Acierno, P Lázpita, and J Toman are acknowledged for their assistance. Research funding was provided by the Mascaro Center for Sustainable Innovation.
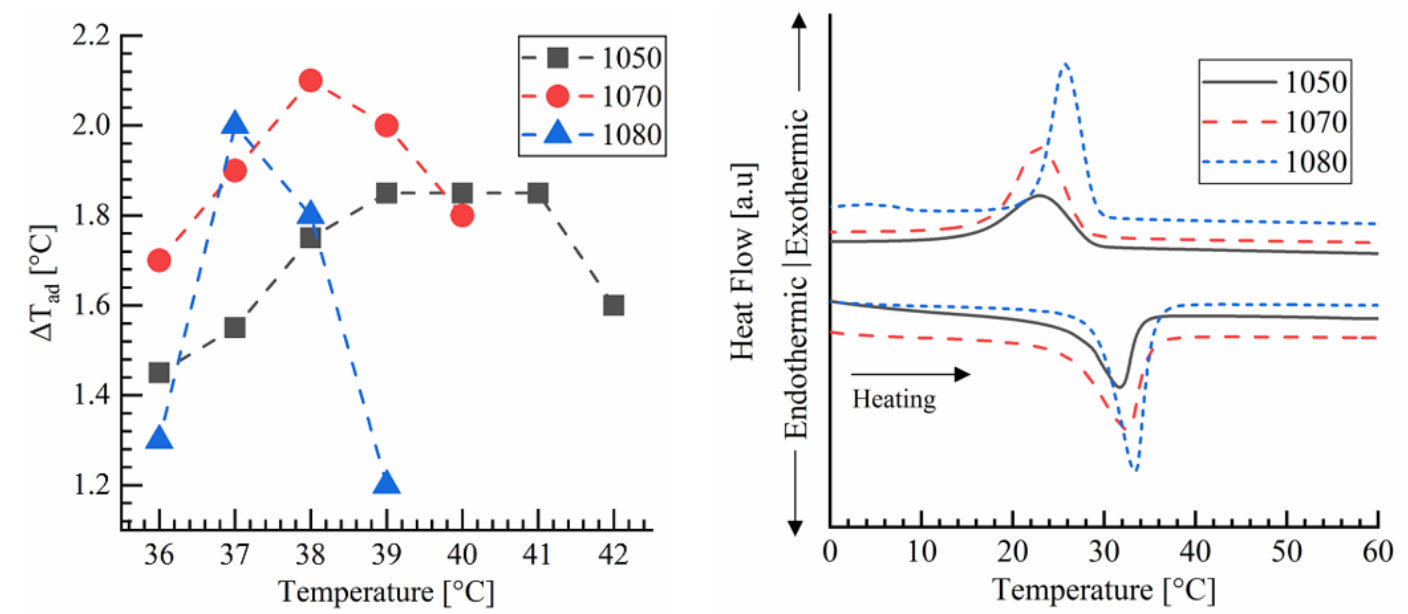

Figure 1. $\Delta \mathrm{T}_{\mathrm{ad}}$ vs. temperature on cooling in a $2 \mathrm{~T}$ magnetic field change for samples sintered at 1050 ${ }^{\circ} \mathrm{C}, 1070{ }^{\circ} \mathrm{C}$, and $1080{ }^{\circ} \mathrm{C}$ (left), and DSC transformation behaviour at zero field (right).

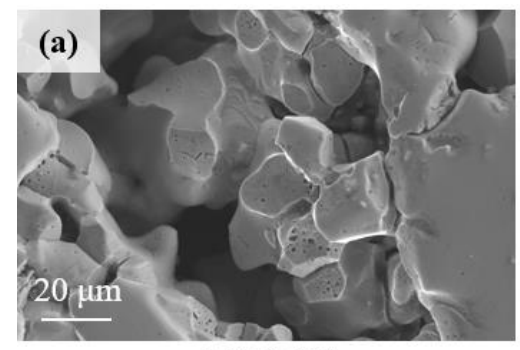

$1050{ }^{\circ} \mathrm{C}$

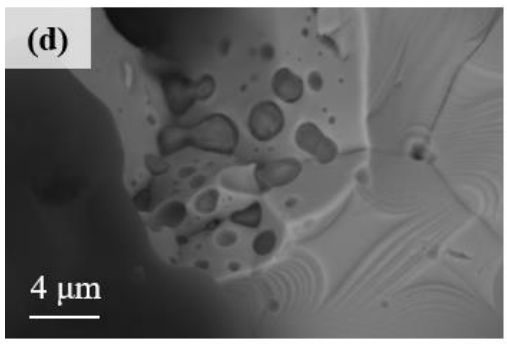

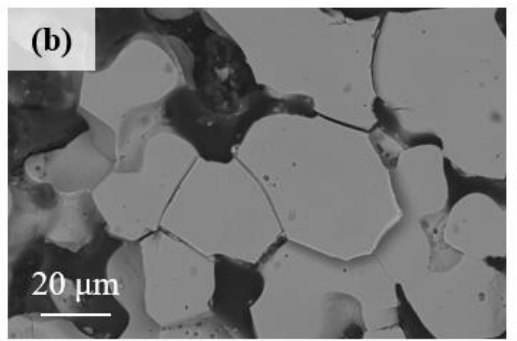

$1070^{\circ} \mathrm{C}$

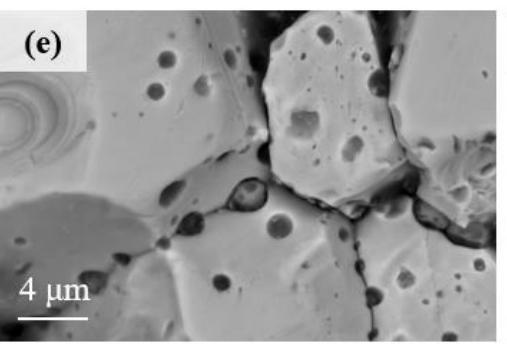

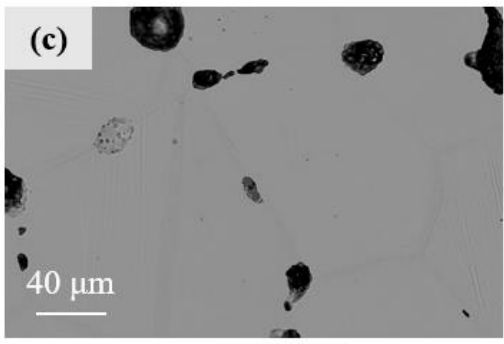

$1080^{\circ} \mathrm{C}$

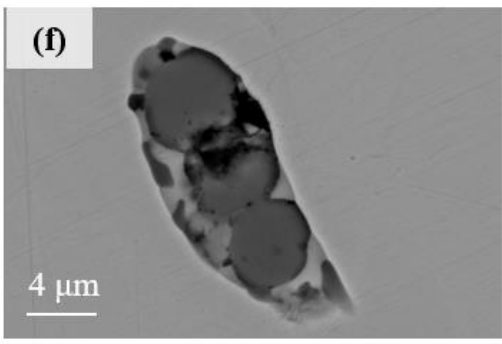

Figure 2. (a-c) Low magnification views of microstructures, showing spotted regions where necks formed and powder particles broke away as well as in remnant porosity. (d-f) High magnification micrographs of embedded $\mathrm{Mn}-\mathrm{O}$ particles at grain boundaries and in remnant porosity. 